\title{
Ueber den Ausdruck des Tetraeders durch die Co- ordinaten der Eckpunkte.
}

\author{
(Von Herrn R. Baltzer in Giessen.)
}

(Aus den Berichten der mathem.-phys. Classe der Sächs. Gesellschaft der Wissenschaften rom 4. Mai 1870.)

Der Ausdruck des Tetraeder-Volums durch die Coordinaten der Eckpunkte wurde zuerst von Lagrange in der Abhandlung über die Pyramiden durch eine Rechnung gefunden, welche ausserhalb ihres Zusammenhanges etwas umständlich ist. Man gelangt zu demselben Ausdruck mittelst der Gleichung für die Ebene, welche 3 gegebene Punkte enthält, wie Salmon Geom. of 3 dimensions 31 gezeigt hat. Derselbe Ausdruck wurde von Monge (J. de l'école polyt. Cah. 15 p. 68) durch Berechnung von prismatischen Segmenten abgeleitet, eine Betrachtung, die in Magnus Sammlung (anal. Geometrie des Raumes \$14) wiedergegeben worden ist. Eine rein geometrische Ableitung der entsprechenden Formel findet man in Möbius Statik $\$$ 64; eine algebraische Ableitung, die auf Multiplication von Determinanten beruht, habe ich (Determ. 5. 15) gegeben; eine andere Ableitung enthält Hesses anal. Geometrie des Raumes (1. Vorlesung). Eine einfache und für die Präliminarien der Raumgeometrie geeignete Ableitung ergiebt sich durch die folgende Betrachtung.

Auch dem Zeichen nach ist $3 O A B C=O A B . N C$, wenn $N C$ den $A b-$ stand des Punktes $C$ von der Ebene $O A B$ bedeutet. In Bezug auf 3 durch $O$ beliebig gezogene Axen habe $A$ die Coordinaten $x_{1}, y_{1}, z_{1}$, u. s. w. Durch Projection der aus den Coordinaten von $C$ bestehenden gebrochenen Linie auf die Normale $n$ der Ebene $O A B$ findet man

$$
N C=x_{3} \cos x n+y_{3} \cos y n+z_{3} \cos z n \text {. }
$$

Ferner werden die Fläche $O A B$ und ihre Projectionen parallel mit $x, y, z$ auf die Ebenen $y z, z x, x y$ durch $p, p_{x}, p_{y}, p_{z}$ bezeichnet, die Normalen der Ebenen $y z, z x, x y$ durch $x^{\prime}, y^{\prime}, z^{\prime}$. Dann hat der Normalschnitt des Prisma, welches die Fläche $O A B$ parallel mit $x$ auf die Ebene $y z$ projicirt, die Werthe

$$
p \cos x n=p_{x} \cos x x^{\prime}
$$

u. s. w. Nun hat das Parallelepiped, dessen Kanten auf $x, y, z$ positive Einheiten sind, die Werthe

$$
\sin y z \cos x x^{\prime}=\sin z x \cos y y^{\prime}=\sin x y \cos z z^{\prime}=\sin x y z ;
$$


folglich ist

$$
2 p \cos x n=\frac{2 p_{x}}{\sin y z} \sin x y z=\left|\begin{array}{ll}
y_{1} & y_{2} \\
z_{1} & z_{2}
\end{array}\right| \sin x y z
$$

u.s.w. Durch Vereinigung der 3 Determinanten findet man

$$
60 A B C=\left|\begin{array}{lll}
x_{1} & x_{2} & x_{3} \\
y_{1} & y_{2} & y_{3} \\
z_{1} & z_{2} & z_{3}
\end{array}\right| \sin x y z
$$

Wenn man die Geraden, auf denen die Kanten $O A, O B, O C$ liegen, durch $f, g, h$ bezeichnet, so ist

$$
6 O A B C=O A . O B . O C \sin f g h \text {. }
$$

Demnach erhält man unter Voraussetzung eines orthogonalen Systems, bei $\operatorname{dem} \sin x y z=1, x_{1}=0 A \cos x f$, u. s. w. ist,-die Gausssche Gleichung

$$
\sin f g h=\left|\begin{array}{lll}
\cos x f & \cos x g & \cos x h \\
\cos y f & \cos y g & \cos y h \\
\cos z f & \cos z g & \cos z h
\end{array}\right|,
$$

ferner durch Multiplication die Staudtsche Gleichung

$$
\begin{gathered}
\sin f g h \sin f^{\prime} g^{\prime} h^{\prime}=\left|\begin{array}{lll}
\cos x f & \cos x g & \cos x h \\
\cos y f & \cos y g & \cos y h \\
\cos z f & \cos z g & \cos x h
\end{array}\right|\left|\begin{array}{ccc}
\cos x f^{\prime} & \cos x g^{\prime} & \cos x h^{\prime} \\
\cos y f^{\prime} & \cos y g^{\prime} & \cos y h^{\prime} \\
\cos z f^{\prime} & \cos z g^{\prime} & \cos z h^{\prime}
\end{array}\right| \\
=\left|\begin{array}{lll}
\cos f f^{\prime} & \cos f g^{\prime} & \cos f h^{\prime} \\
\cos g f^{\prime} & \cos g g^{\prime} & \cos g h^{\prime} \\
\cos h f^{\prime} & \cos h g^{\prime} & \cos h h^{\prime}
\end{array}\right|
\end{gathered}
$$

und durch Vereinigung der beiden Systeme

$$
\sin ^{2} f g h=\left|\begin{array}{ccc}
1 & \cos f g & \cos f h \\
\cos f g & 1 & \cos g h \\
\cos f h & \cos g h & 1
\end{array}\right| .
$$

